\title{
Observation of Bone Tissue Metabolism and Bacterial Biofilm in Aqueous Solution Using ASEM
}

\author{
Chikara Sato ${ }^{1}$, Masami Naya ${ }^{1}$, Memtily Nassirhadjy ${ }^{2}$, Mari Sato ${ }^{1}$, Naoki Kasahata ${ }^{1}$ and Shinya \\ Sugimoto $^{3}$ \\ ${ }^{1}$ National Institute of Advanced Industrial Science and Technology (AIST), Tsukuba, Ibaraki, Japan, \\ ${ }^{2}$ Xinjiang Medical University, Urumqi, Xinjiang, China (People's Republic), ${ }^{3}$ The Jikei University School \\ of Medicine, Shimbashi, Tokyo, Japan
}

The ASEM ClairScope ${ }^{\mathrm{TM}}$ (JEOL Ltd.) is an inverted scanning electron microscope (SEM) (Fig. 1 left), which realizes SEM of a sample at atmospheric pressure in a readily-accessible, open container called the ASEM dish [1]. The dish has eight $0.25 \mathrm{~mm} \times 0.25 \mathrm{~mm}, 100 \mathrm{~nm}$-thick SiN film windows and can hold a few milliliters of culture medium for cells. It allows various types of aldehyde-fixed cells and tissues (Fig. 1 right) to be quickly observed in radical scavenger glucose solution at high resolution [1]. An optical microscope (OM) positioned above the ASEM dish can be used to observe wide areas of the sample from above, and the SEM to image specified smaller regions from below through the SiN film in the base of the dish (Fig. 1 left). The optical axes of both microscopes are aligned to image the same area in the center, and the specimen stage can be moved two-dimensionally $(\mathrm{x}-\mathrm{y})$ for targeting. The resolution measured between gold particles near the SiN film was 8nm.

The lungs are known to be the organs easily metastasized by cancer. OM is usually employed to make the diagnosis. However, it takes more than 15 minutes for cryo-thinsectioning. Comparison of lung tissue excised from normal mice and from mice with tumors by ASEM suggests that the ASEM could become an important less time-consuming diagnostic tool [2]. ASEM of 200 micrometer-thick normal lung tissue revealed typical thin-wall structures with alveoli, alveolar ducts, a vein system and trachea (Fig. 1b), but only faint traces of the regular alveoli and alveolar ducts were discernable for metastasized lung (Fig. 1c) [2]. Nuclei close to the surface of the tissue slabs appeared as bright cores and were larger in the breast cancer cells with a different shape. Biofilms formed by MRSA becteria on ASEM dish were stained using OA/UA/LC solutions and imaged [3]. It revealed small spheres and fibrils, which might be secreted vesicles and intercellular membraneous connections (Fig. 1d).

To image osteoclasts involved in osteoporosis, spongy bone tissue was immuno-labelled for the osteoclast marker cathepsin K and imaged by ASEM [4]. Cathepsin K protease secreted by osteoclasts digests bone collagen and thus plays a critical role in bone absorption. Femur slabs, 200 microm thick, were labeled with anti-cathepsin K antibody and further with a secondary antibody conjugated to FluoroNanogold, gold-enhanced, and counter-stained with PTA. ASEM images showed that trabeculae in the spongy bone were surrounded by and attached to many cells of various shape (Fig. 2). Higher magnification posturated that large cells attached to the thin trabecula walls had many small bright dots, i.e., gold particles, revealing that these cathepsin K positive cells are osteoclasts (Fig. 2b-d arrowheads). Some of the cathepsin Kpositive cells covered a pit of thin mouse trabeculae, and many probably secreted and/or digested substances were observed in the pit (Fig. 2c). The trabeculae themselves were also sometimes partly immuno-labelled (arrow), indicating that cathepsin K connects with bone. 

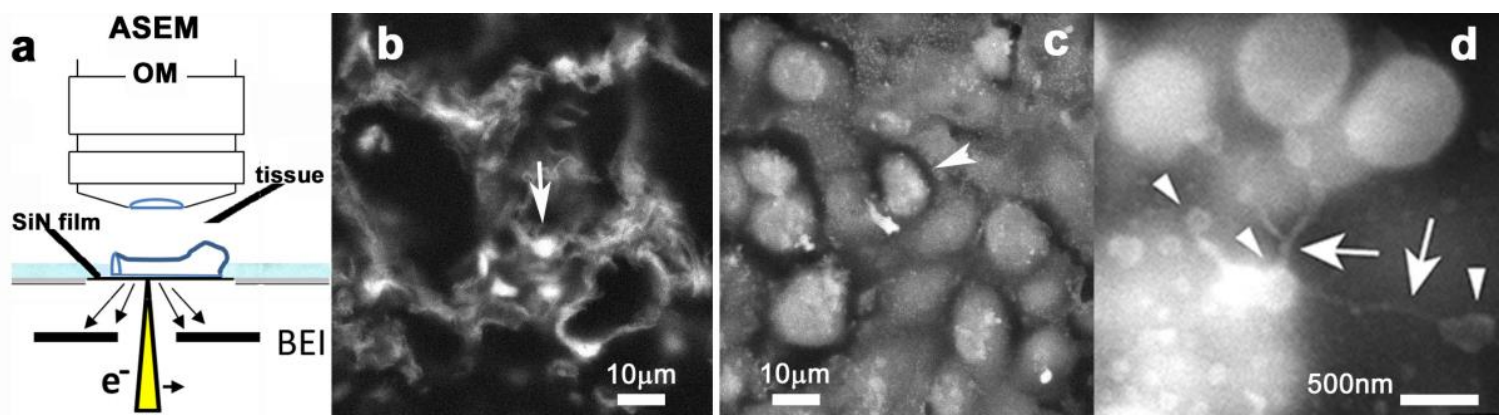

Figure 1. (a) ASEM system used to image aldehyde-fixed cells and tissues immersed in radical scavenger, dextrose solution. An optical microscope (OM) is suited above the inverted SEM, with the 2-3 ml-capacity specimen dish in between. This detachable, 35-mm ASEM dish has eight 100-nm thick $(250 \times 250)-\mu \mathrm{m}$ $\mathrm{SiN}$ film windows. The observable specimen thickness from the $\mathrm{SiN}$ film was measured to be 2-3 microm at an acceleration voltage of $30 \mathrm{kV}$. (b) ASEM image of normal lung tissue stained with platinum-blue (Pt-blue) and PTA. Alveoli and alveolar ducts are imaged with normal sized nuclei (arrow). (c) Comparative imaging of tissue excised from a lung metastasized by breast cancer cells, that have a different shape and their nuclei are larger (arrowhead) [2]. (d) Biofilm formed by MRSA on ASEM dish [3].
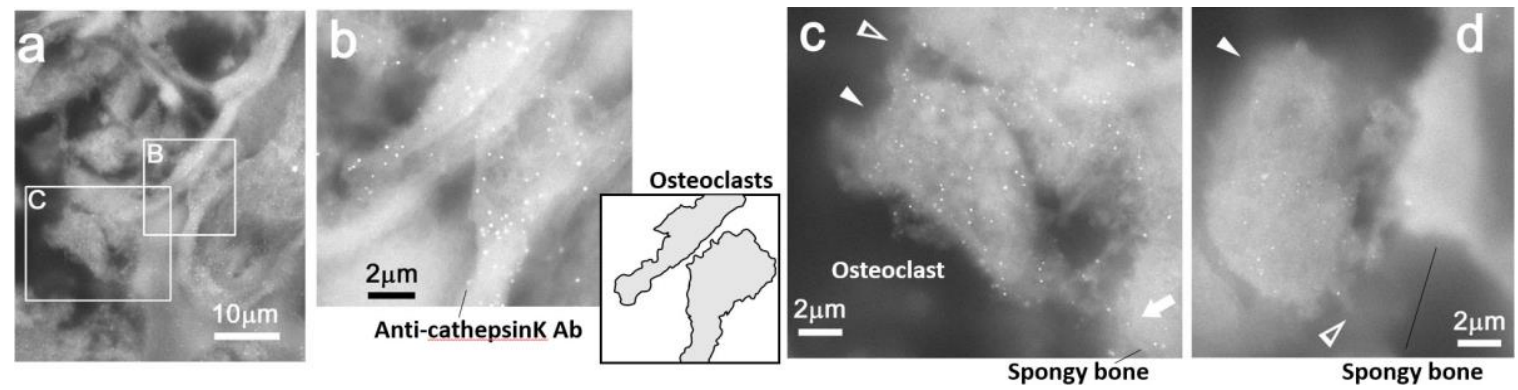

Figure 2. Distribution of cathepsin $\mathrm{K}$ in femur spongy bone in solution using immuno-gold labeling and ASEM. Femur thick slabs were labeled with anti-cathepsin K antibody and further with FluoroNanogoldconjugated secondary antibody, gold-enhanced, and stained with PTA. (a) ASEM image of trabeculae of spongy bone. (b-d) Higher magnification of (a). (c) A cathepsin K positive cell attached to a thin trabecula. Gold signals are imaged as strong bright dots (arrow). (d) Another area observed at higher magnification indicates that cathepsin $\mathrm{K}$ positive cell (arrowheads) covers a pit of thin trabeculae. Many blobby substances were observed between the cell and the trabecula [4].

\section{References}

[1] H. Nishiyama, et al, J Struct Biol, 172 (2010) 191-202.

[2] N. Memtily, et al, Int J Oncol, 46 (2015) 1872-1882.

[3] S. Sugimoto, et al, Sci Rep, 6 (2016) 25889.

[4] C. Sato, et al, Sci Rep, 9 (2019). 